“ (C) 2013 IEEE. Personal use of this material is permitted. Permission from IEEE must be obtained for all other uses, in any current or future media, including

reprinting/republishing this material for advertising or promotional purposes, creating new collective works, for resale or redistribution to servers or lists, or reuse of any copyrighted component of this work in other works." 


\title{
Developing an Application Based on OpenFlow to Enhance Mobile IP Networks
}

\author{
Pakawat Pupatwibul, Ameen Banjar, Abdallah AL Sabbagh, and Robin Braun \\ Centre for Real-Time Information Networks (CRIN) \\ University of Technology, Sydney \\ Sydney, Australia \\ ndomon66@gmail.com,dr.banjar@gmail.com, asabbagh@eng.uts.edu.au, robin.braun@uts.edu.au
}

\begin{abstract}
Mobile IP has been designed to maintain permanent IP address for mobile users while they are moving from one point to another. It allows the mobile node to use two IP addresses: a static home address and a care-of address, which changes at each point of re-attachment. However, Mobile IP requires a location update message to be sent to home agent for each new connection. This will potentially increase the handoff latency and leads to high load on the global Internet. This paper presents the concepts and the challenges of Mobile IP networks and then proposes the use of OpenFlow approach as an alternate transport mechanism to perform routing and to provide network connectivity for Mobile IP networks. The proposed application determines calculations and reroutes the subsequent packets. OpenFlow aims to optimize routing path and handoff performance by using controller's application and exchanges controllers' information.
\end{abstract}

Index Terms-Mobile IP; OpenFlow; Network Mobility; Software Defined Networking; Next Generation Networks.

\section{INTRODUCTION}

Due the increase of connected mobile nodes, next generation networks will require scalable and maintainable connections for ever changing environment. These connections need to ensure that mobile devices can be attached to the Internet even as they move from one place to other, by establishing new links and replacing previous links. For these reasons, Mobile IP has been developed as a solution of moving devices and to provide better experience services, using an alternative IP address that offers a short routing path.

However, one fundamental concept of Mobile IP is that the mobile device is always attached to its home network, which is considered as a limitation of the Mobile IP approach. Therefore, when the mobile device moves to another network, it can cause a number of challenges such as security, triangular connection, and handoff latency [1]. For example, when using tunneling, in case if that tunnel is broken, the transmission of packets could go to a malicious node. In addition, the Mobile IP routes the packets to the home network every time. This will cause load traffic and select unsuitable routing path. Moreover, the Mobile IP considers the distance between home agent and the foreign agent which cause handoff latency [2]. Some one can argue that, if the mobile node travels from home network to a foreign network, which is located just near to the sender, the Mobile IP needs to use a triangle routing path.

OpenFlow is an emerging network paradigm which decouples the control path from the data path of forwarding elements. Using this strategy, OpenFlow allows network elements such as routers or switches to be programmable via a standardized interface [3]. In addition, OpenFlow provides high flexibility of novel packet forwarding and routing of network flows that can be adapted to the ever changing environment by using virtualization and flow-based routing.

Previous issues are surrounding the mobile nodes and the Mobile IP is insufficient for long/short connection with mobile device for appropriate transmission of flows. Therefore, this paper introduces OpenFlow as a base to support alternative IP address for mobile node. OpenFlow can manage the security of transition process and can manage authentication of users. In addition, OpenFlow can optimize the routing process, where it can deliver the packets directly to a certain OpenFlow switch which hold the mobile node. The correspondent node does not require a detour by passing the home network anymore. Using OpenFlow allows the constructing of flow entries within OpenFlow switch to update the new address of the mobile node. Thus, when the source has packets that need to be sent to a mobile node, the controller knows how to update, convert and then send that packets with the best routing path.

This article follows the logical outline indicated as following. Section II presents the overview of Mobile IP protocol. In Section III, the limitations of Mobile IP are described. Section IV addresses the main factors that influence the need of OpenFlow as a base for Mobile IP. In Section V, the implementation of OpenFlow based for Mobile IP is proposed. Finally, Section VI summarizes and concludes the paper.

\section{MOBILE IP}

With the rapid growth of wireless communications and the increase of market demands, it is inevitable to develop and introduce the Mobile IP technology. Mobile IP enables a mobile node to maintain active connections with the same IP address when it traverses from place to place in the Internet. It adopts the existing IP protocol to implement the communication effectively for the mobile nodes [4], [5], [6], 
[7]. It is necessary to frame the discussion with few terminologies in order to understand the operation of Mobile IP. According to the mobile IP specification, it introduces the following new functional entities:

Mobile node: A host or router that changes its place of connection from one network to another. A mobile node may change the location without changing its IP address or may continue to communicate to other Internet nodes at any location using its (constant) IP address.

Home address: An IP address that is allocated for an extended period of time to a mobile node. This address will remain unchanged regardless of where the node is connected to the Internet.

Home agent: A router on a mobile node's home network which tunnels datagrams for delivery to the mobile node when it is away from home, and maintains current location information for each.

Home network: A possibly virtual network, having a network prefix matching that of a mobile node's home address. Note that standard IP routing mechanisms will deliver datagrams destined to a mobile node's Home Address to the mobile node's Home Network.

Foreign agent: A router on a mobile node's visited network which cooperates with the Home Agent to complete the delivery of datagrams to the mobile node while it is away from home.

Foreign network: Any network other than the mobile node's Home Network.

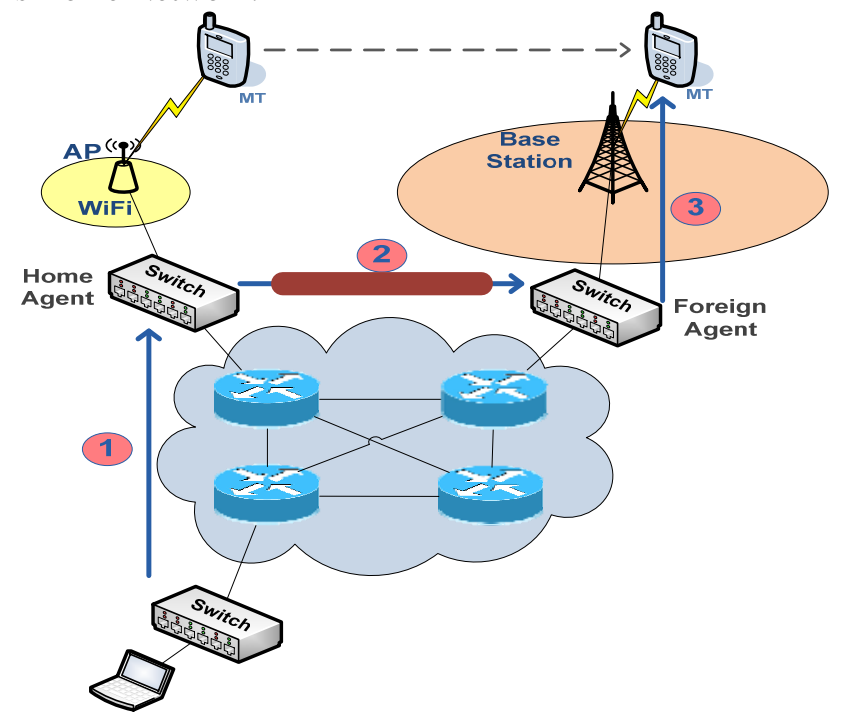

Fig. 1. Mobile IP Scenario.

Care-of address: The termination point of a tunnel toward a mobile node, for datagrams forwarded to the mobile node while it is away from home. There are two different types of care-of address: (1) foreign agent care-of address - an address of a foreign agent with which the mobile node is registered; (2) collocated care-of address - an externally obtained local address which the mobile node has associated with one of its own network interfaces.
Correspondent node: A peer with which a mobile node is communicating. A correspondent node may be either stationary or mobile.

Tunnel: The path followed by a datagram while it is encapsulated. The model is that, while encapsulated, a datagram is routed to a knowledgeable agent, which decapsulates the datagram and then forwards it along to its ultimate destination.

Figure 1 shows a general scenario on how Mobile IP can cope with the dynamic IP addresses. A mobile node is allocated to a specific network so called home network. Its IP address on that network is static, known as its home address. When a mobile node traverses to another attachment point that belongs to a different network, that network is considered as a foreign network. After the mobile node is reconnected, it needs to be registered to a network node, typically a router on the foreign network known as a foreign agent. Then the mobile node communicates with a similar agent called home agent on the user's home network and gives the home agent the care-of address of the mobile node. This address determines the foreign agent's location. Basically, one or more routers on a network can implement the roles of both home and foreign agents.

The following steps will demonstrate an outline when IP datagrams are exchanged over a connection between mobile node and another host (Correspondent Node). Figure 1 may be used to help envision the roles played by the entities.

1. CN transmits an IP datagram to the designation of mobile node (MT), with MT's home address in the IP header. IP datagram is routed to MT's home network.

2. At home network, the home agent will intercept the incoming IP datagram, encapsulate it inside a new IP datagram that has the MT's care-of address in the header, and then retransmits the datagram. Using outer IP datagram with a different destination IP is known as tunneling. Finally, this IP datagram is routed to the foreign agent.

3. The foreign agent takes off the outer IP header, encapsulates the original IP datagram and delivers it to MT across the foreign network.

4. When MT forwards traffic back to CN, it uses CN's IP address. In this case is a fixed address as $\mathrm{CN}$ is not a mobile node. Each IP datagram is forward by MT to a router on the foreign network for routing to $\mathrm{CN}$.

5. The IP datagram from MT to $\mathrm{CN}$ travels directly across the Internet to CN, using CN's IP address.

Mobile IP can be thought of as a way of performing three related functions: (1) Discovery - there is an agent discovery mechanism defined, so that mobile nodes can identify their prospective home agents and foreign agents as they move from place to place within the Internet; (2) Registration - a mobile node uses an authenticated registration procedure to inform its home agent of its care-of address; (3) Tunneling - Mobile IP defines a simple mechanism to deliver datagrams to the mobile node when it is away from its home network. Tunneling is used 
to forward IP datagrams from a home address to a care-of address [8], [9].

\section{MOBILE IP CHALLENGES}

Mobile IP provides capability of changing the network attachment of the users without losing their connection while users moving. However, there are some issues should be considered to enhance services. These issues are presented in this section as following:

\section{A. Security issues}

Security is considered as a main challenging process with in Mobile IP network. The mobility of communication devices and wireless channel introduce some security issues. Security issues for Mobile IP are considered when the mobile device registers the new care-of address to the home agent, this registration messages requires an authentication to improve the security performance [10], [11]. Then, after registration some one can attack the session to transfer the data to a malicious node.

\section{B. Triangle routing issues}

Mobile IP scheme is based on triangle routing, where corresponding node sends packet to the mobile node's home network. Then the home agent sends packets to the foreign agent using IP-IP tunnelling [12]. After that the mobile node sends packets directly to the corresponding node. The IP-IP tunnelling can cause long delays and also unnecessary network congestion. In addition, the tunnelling will consume an extra bandwidth, from the home agent to the care-of address [13].

\section{Handoff issues}

Handoffs usually cause packets loss, which is especially harmful to applications, such as video streaming. Whereas, the home agent is responsible of handoff, it makes lots of signalling traffic between the mobile node and the home agent, which lead to network congestion. Moreover, simultaneous handoffs for number of mobile nodes within wide area networks WAN can result network congestion [14].

\section{The NeEd For OpenFlow-BASED MOBILE IP}

As Mobile IP has some limitations mentioned above, and therefore one can argue that it cannot be feasible. The main factors that influence the implementation of mobile IP based on OpenFlow networks are discussed in this section.

\section{A. Mobility support}

Figure 2 shows the global forecast for total mobile devices released February 2012. It has been shown that the forecasted number of mobile devices is predicted to increasing from 1.03 billion in 2011, or 10.1 percent of total fixed mobile devices, to 7.6 billion by the year 2016. This trend of wireless communications has been the driving motivation for the creation of new protocols as well as increased the needs for mobility support. As a result, it would be one of the most promising reasons for implementing Mobile IP based on OpenFlow networks.

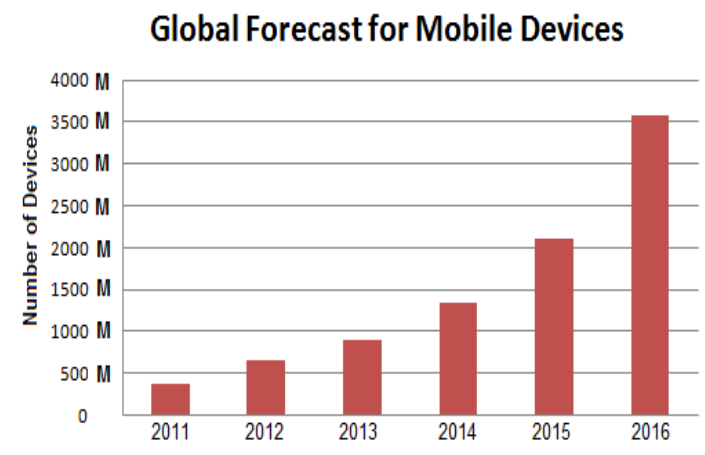

Fig. 2. Global forecast for mobile devices. Data presented from Ref [15].

\section{B. Alternative technologies}

In Mobile IP, a mobile node discovers its Foreign and Home Agents during agent discovery. Then the mobile node makes its presence by registering the current location with the Foreign Agent and Home Agent. Finally, tunnelling is set up by the Home Agent to the care-of address (current location of the mobile node on the foreign network) to route packets to the mobile node as it roams [16]. One possible alternative is that Mobile IP based OpenFlow can compute the best routes without tunnelling process. The controller will calculate and install the flow tables' entries for new destination address whenever it changes its point of attachment. For example, packet moves from Flow Table 1 to Flow Table 4 directly (see Figure 3). This would turn out to be a compelling technique for providing seamless mobility support for the end-users.
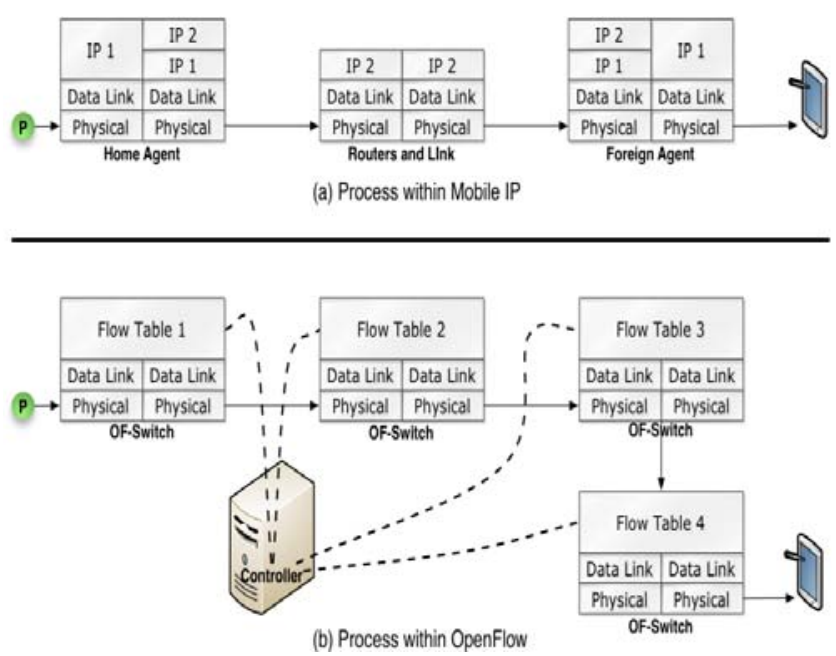

Fig. 3. Comparison of traditional Mobile IP and proposed OpenFlow based for Mobile IP. 


\section{Improved Handoffs process}

Currently, handover between different wireless technologies is hard to manage. This is because the intermediate links between the core networks are different, as they are built around a single wireless technology. By deploying OpenFlow based Mobile IP, Figure 3 shows that handover process can be achieved in a simple way of local registration to the new OpenFlow switch which informs the controller regarding new location. OpenFlow based Mobile IP can provide a simple hand-off solution that will allow mobile nodes device to move around seamlessly within a larger network, comprised of many connection points, without once losing their connection.

\section{MOBILE IP BASED ON OPENFLOW IMPLEMENTATION}

In regards to technologically optimization, OpenFlow based Mobile IP will require minor changes to the operating systems of the correspondent nodes and will also be feasible with the specifications of the L2 switching suite. The proposed development of Mobile IP based on OpenFlow networks is introduced in this section.

\section{A. Registration process}

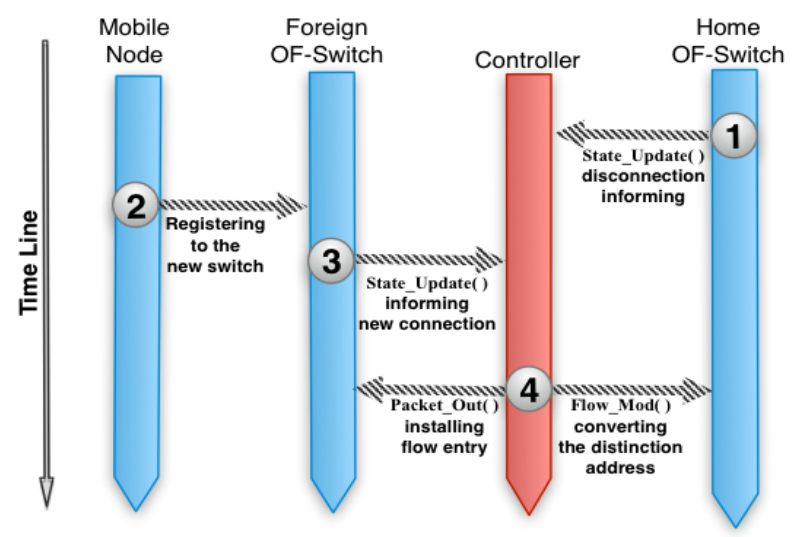

Fig. 4. Exchanging messages over the time.

OpenFlow-based Mobile IP can introduce minimal overhead for delivering the data and signalling information as well as effective routing scheme by using direct routes between the mobile node and the correspondent node. Moreover, the handoffs process will be performed with minimal packet loss due to the localized handling of the handoffs are performed somewhat faster as the network delay between mobile node and home switch does not affect the handoff.

Deploying Mobile IP in OpenFlow networks can enhance the mobility of mobile nodes where routing optimization and the high level decision making are performed by the controller. Moreover, Home agent and Foreign agent will be replaced by OpenFlow controller application. In Mobile IP, all packets sent to the mobile node must pass through the home agent, but the route may not be the best.

In OpenFlow based Mobile IP, after mobile node moves from Home switch to the Foreign switch, according to Figure 4, it registers to the new switch basically Foreign switch. Then both Home and Foreign switch notifies the controller, using
State_Update(), for new information regarding disconnection and reconnection. After that, the controller is able to calculate and install new flow entries in both switches. The controller uses Flow_Mod(), to modify the flow entry and convert the destination IP for Home switch. Also, the controller uses Packet_Out() for Foreign switch to guide the packet to reach its destination mobile node. In addition, each switch of OpenFlow is responsible for informing the controller, every time a mobile node moves. The Foreign switch informs for new connection and the Home switch updates for disconnection.

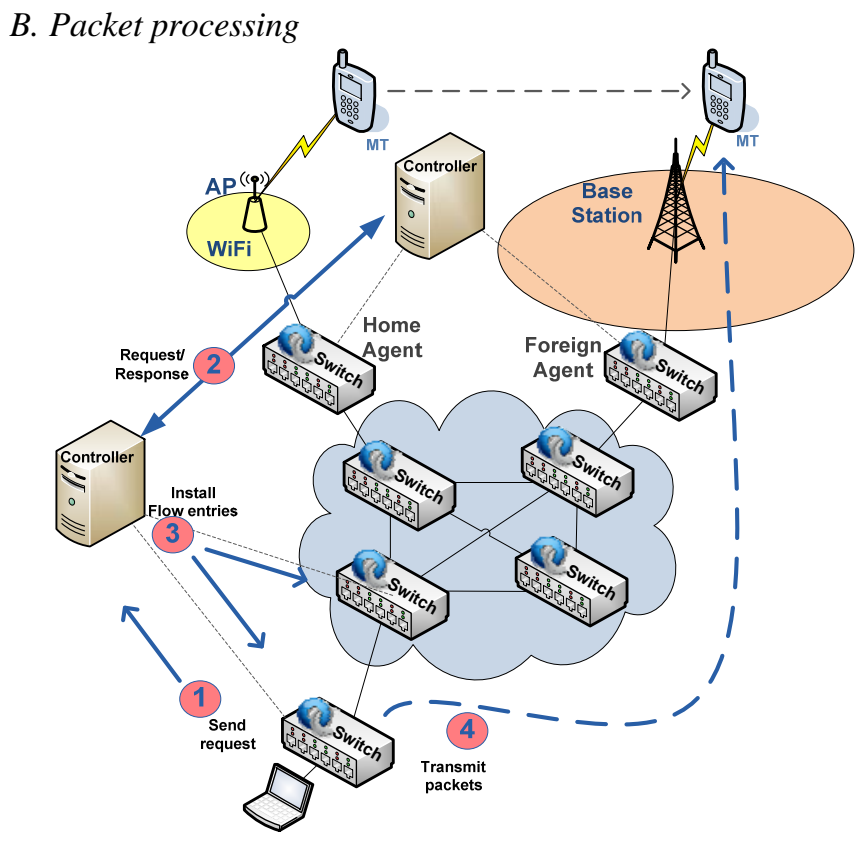

Fig. 5. OpenFlow base for Mobile IP scenarios.

Figure 5 illustrates the corresponding packet processes after the mobile node moves. Firstly, the $\mathrm{CN}$ sends the subsequence packet to the attached switch where the mobile node has been moved as shown in Figure 4. The controller which manages the CN's switch receives a request. Then the controller will check its library looking for the IP address of the mobile node. After that, the controller sends and receives new information from the other controller which manages the Foreign switch. Therefore, the controller now can update all the corresponding switches and the packets will be transmitted to the destination. In more details, the CN's controller will computes the new destination address and installs flows to the Foreign switch for the transparent transmission.

The information is transferred via a definite port number using a secure TLS channel. If the mobile node moves again, the previous Foreign switch will transfer the updated information to the controller to ensure that the packets are transferred to the new Foreign switch.

Meanwhile the controller will also update information and again install flows to this new Foreign switch so the subsequent packets will be transferred directly from the correspondent node. This method of delivering packets to mobile node within OpenFlow switches can help to reduce the time delay for re- 
registration and improve the handoff performance as well as solving the problems of triangle routing.

\section{Mobile IP's Controllers Application}

The Mobile IP's controller application is presented below with the details on the algorithm of this application. The OpenFlow controller is enhanced to calculate and install the flow entries for all corresponding OpenFlow switches. For Home switch, it converts the destination IP and installs the flow entries along to chosen path until it reaches the last careof address. Meanwhile Foreign switch is responsible for informing and updating the controller for registration of the mobile node.

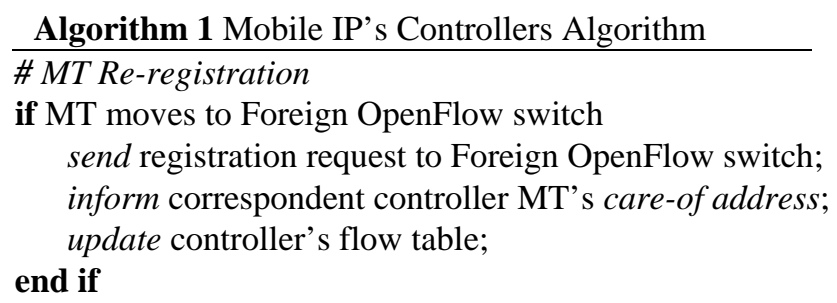

\section{CONCLUSION}

In this paper, we have discussed most of the fundamental features of mobile IP, an extension standard protocol to IP which allows mobile nodes to move seamlessly from place to place within the Internet. It has been evident in this paper that even with some limitations in the implementation of Mobile IP, there will be an increased demand for Mobile IP in the future.
This paper has also discussed the needs for a successful deployment of Mobile IP. In this regard, we propose OpenFlow networks as an alternative technology to develop Mobile IP with the hope to address the limitations that are currently present in Mobile IP. By using OpenFlow based Mobile IP, routing of packets and handover performance are optimized by the controllers' application. This will improve mobile users experience while maintaining seamless pervasive connectivity. Future work can be an implementation of the designed algorithm and a run of experiments using Omnet++, focusing on wireless mobile applications.

\section{ACKNOWLEDGMENT}

This work is sponsored by the Centre for Real-Time Information Networks (CRIN) in the Faculty of Engineering \& Information Technology at the University of Technology, Sydney (UTS).

\section{REFERENCES}

[1] C. E. Perkins, "Mobile IP," Communications Magazine, IEEE, vol. 35, pp. 84-99, 1997.

[2] J. Chandrasekaran, "Mobile IP: Issues, Challenges and Solutions," Master's thesis, Department of Electrical and Computer Engineering Rutgers University, 2009.

[3] N. McKeown, et al., "OpenFlow: enabling innovation in campus networks," ACM SIGCOMM Computer Communication Review, vol. 38, pp. 69-74, 2008.

[4] Rfc 3344 - IP mobility support for ipv4. http://tools.ietf.org/html/rfc3344.

[5] Rfc 4721 - mobile IPv4 challenge/response extensions (revised). http://tools.ietf.org/html/rfc4721.

[6] Introduction to Mobile IP. http://www.hpl.hp.com/personal/Jean Tourrilhes/MobileIP/ppal.html.

[7] Mobile-IP: Transparent host migration on the internet. http://www.linuxjournal.com/article/1271.

[8] C. Perkins, ed., "IPv4 Mobility Support," RFC 2002, Oct. 1996.

[9] C. E. Perkins, "Mobile networking through mobile IP," Internet Computing, IEEE, vol. 2, pp. 58-69, 1998.

[10] A. Habib, et al., "Detecting service violations and DoS attacks," in Proceedings of 2003 Internet Society Symposium on Network and Distributed System Security (NDSS’03), 2003.

[11] H. Petander, Mobile IP Route Optimization, White Paper, Helsinki University of Technology, May 2000.

[12] J. Redi and P. Bahl. Mobile IP: a solution for transparent seamless mobile computer communications. In Report on Upcoming Trends in Mobile Computing and Communications.

[13] A. G. Valkó, "Cellular IP: a new approach to internet host mobility," ACM SIGCOMM Computer Communication Review, vol. 29, pp. 50-65, 1999.

[14] Ye Minhua, Liu Yu, Zhang Huimin. Handover Technology in Mobile IP [J]. Telecommunication Technology, 2003(12).

[15] Cisco ${ }^{\circledR}$ Visual Networking Index (VNI), The Zettabyte Era, White Paper, Cisco VNI:Forecast and Methodology 2011-2016, May 2012.

[16] C. E. Perkins and D. B. Johnson, "Route optimization for mobile IP," Cluster Computing, vol. 1, pp. 161-176, 1998. 